\title{
The Role of Shugoshin in Meiotic Chromosome Segregation
}

\author{
D. Clift A.L. Marston
}

The Wellcome Trust Centre for Cell Biology, School of Biological Sciences, University of Edinburgh, Edinburgh, UK

\author{
Key Words \\ Chromosome Segregation - Cohesin - Meiosis • PP2A • \\ Separase $\cdot$ Shugoshin
}

\begin{abstract}
During meiosis, DNA replication is followed by 2 successive chromosome segregation events, resulting in the production of gametes with a haploid number of chromosomes from a diploid precursor cell. Faithful chromosome segregation in meiosis requires that sister chromatid cohesion is lost from chromosome arms during meiosis I, but retained at centromeric regions until meiosis II. Recent studies have begun to uncover the mechanisms underlying this stepwise loss of cohesion in meiosis and the role of a conserved protein, shugoshin, in regulating this process.
\end{abstract}

Copyright $\odot 2011$ S. Karger AG, Basel

The precise distribution of chromosomes during cell division is essential for the growth and proliferation of all living organisms. Defects in chromosome segregation will inevitably lead to the production of cells containing abnormal numbers of chromosomes, known as aneuploidy, which likely contributes to cancer progression and is a cause of infertility and birth defects [Rajagopalan and Lengauer, 2004; Hassold et al., 2007]. During mitosis, replicated chromosomes (sister chromatids) are segregated away from each other, resulting in the production of

\section{KARGER}

Fax +4161306 1234

E-Mail karger@karger.ch

www.karger.com (c) 2011 S. Karger AG, Basel

Accessible online at: www.karger.com/cgr daughter cells carrying a chromosome complement identical to that of the progenitor cell. Mitotic chromosome segregation requires that sister chromatids attach, via their kinetochores, to microtubules emanating from opposite poles of the spindle (bi-orientation). Cohesion between sister chromatids facilitates the bi-orientation process by counteracting the pulling forces of spindle microtubules, thereby generating tension across kinetochores, which stabilises kinetochore-microtubule attachment. Once sister chromatids are attached and under tension, chromosome segregation is triggered by the complete loss of sister chromatid cohesion [Nasmyth, 2001].

Meiosis is a specialised cell division programme, essential for sexual reproduction, in which 4 daughter cells with a complete haploid genome are produced from a diploid precursor cell. This reduction in chromosome number is achieved by 2 successive chromosome segregation events (meiosis I and meiosis II), which follow a single round of DNA replication (fig. 1). Homologous chromosomes are segregated away from each other in meiosis I, whilst sister chromatid segregation does not occur until meiosis II. There are 3 crucial events that must take place during meiosis I to ensure the accuracy of this reductional chromosome segregation pattern. First, linkages must be established between maternally and paternally derived homologous chromosomes to ensure their attachment to opposite poles of the meiosis I spindle. Most commonly, meiotic recombination provides these linkages by generating at least one chiasma, in which a sister chromatid 


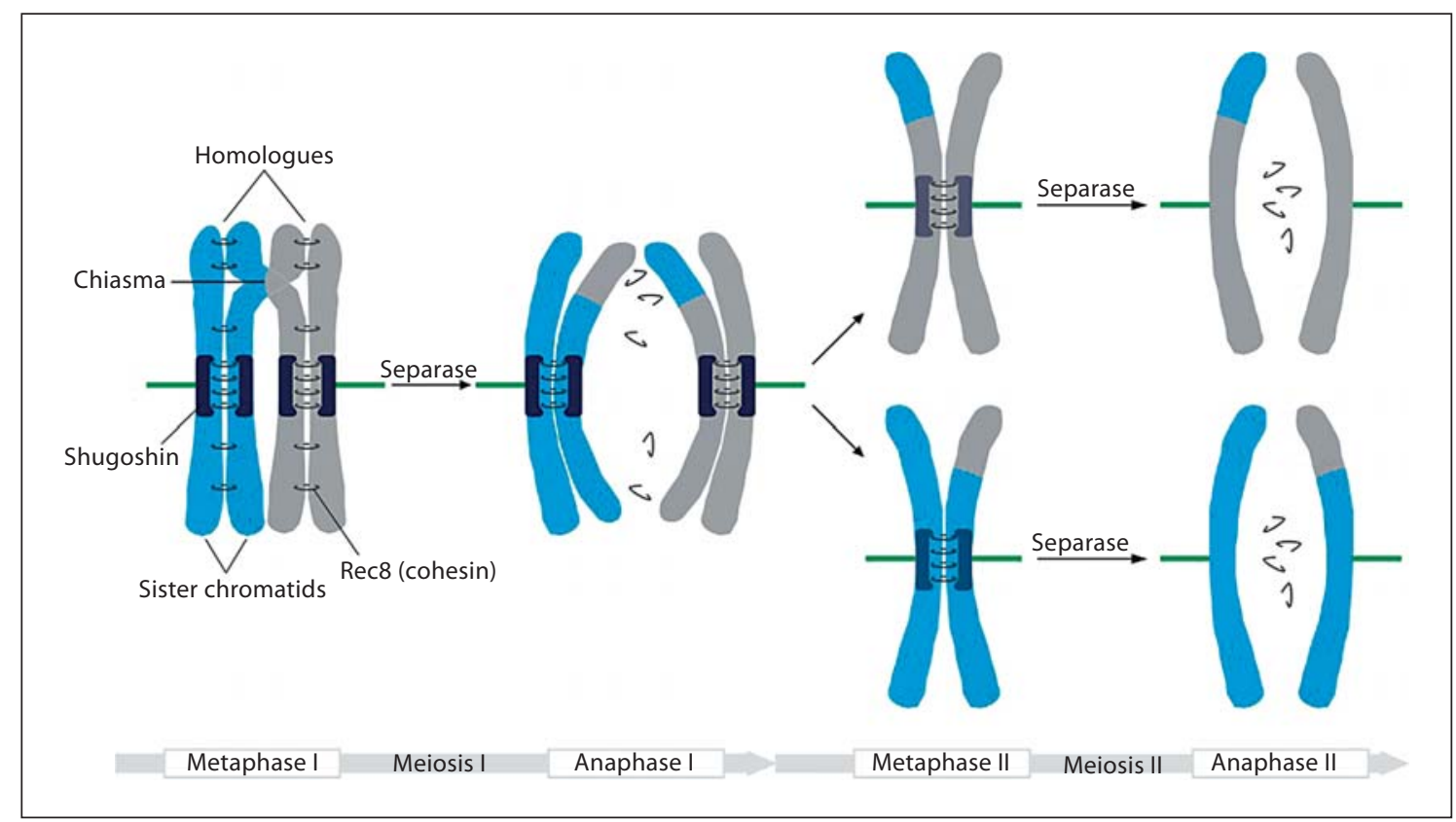

Fig. 1. The stepwise loss of cohesion in meiosis is regulated by shugoshin. During meiosis, sister chromatids are held together by cohesin complexes containing the meiosis-specific Rec8 subunit. In meiosis I, homologous chromosomes are linked by chiasmata and sister kinetochores attach to microtubules emanating from the same spindle pole. Separase cleaves the Rec 8 on chromosome arms; however, $\operatorname{Rec} 8$ at centromeres is protected due to the pres- ence of shugoshin. In meiosis II, the residual centromeric cohesion facilitates the attachment of sister kinetochores to microtubules emanating from opposite poles of the spindle. Tension across sister kinetochores may inactivate shugoshin, and sister chromatid separation is triggered by cleavage of the remaining Rec 8 by separase.

\section{Shugoshin Protects Centromere Cohesion during Meiosis I}

Sister chromatid cohesion is mediated by the multiprotein cohesin complex, which forms a ring-shaped structure that encompasses sister chromatids. The core of the mitotic cohesin complex consists of 4 subunits: $\mathrm{Smcl}$, Smc3, Scc1/Rad21 and Scc3. Mitotic sister chromatid cohesion is destroyed in anaphase owing to the activation of separase (Esp1 in budding yeast; Cut1 in fission yeast), a cysteine protease that cleaves $S c c 1 / \operatorname{Rad} 21$ leading to the opening of the cohesin ring [Nasmyth and Haering, 2009]. During meiosis, however, the $S c c 1 / \operatorname{Rad} 21$ cohesin subunit is largely replaced by a meiosis-specific Rec8 subunit [Klein et al., 1999; Parisi et al., 1999; Watanabe and Nurse, 1999; Pasierbek et al., 2001; Eijpe et al., 2003]. Nonetheless, both the segregation of homologous chromosomes in anaphase I and the segregation of sister chromatids in anaphase II also require cleavage of Rec 8 by separase [Buonomo et al., 2000; Kitajima et al., 2003; Kudo et al., 2006], suggesting that $\operatorname{Rec} 8$ is specifically protected from separase activity at the pericentromere during meiosis I. 
An ingenious genetic screen in fission yeast led to the identification of a conserved protein responsible for Rec8 protection [Kitajima et al., 2004]. Fission yeast cells engineered to produce Rec 8 instead of Scc1/Rad21 during mitotic growth are viable, indicating that factors other than Rec8 itself are responsible for the protection of pericentromeric Rec8 [Watanabe and Nurse, 1999]. Kitajima et al. [2004] screened for genes that when co-overexpressed with Rec8 in mitotic cells allowed cohesin to persist on chromosomes, resulting in a failure to separate sister chromatids and lethality. They isolated one such gene and named the product of this gene shugoshin (Sgol), which means 'guardian spirit' in Japanese [Kitajima et al., 2004]. Independent screens for deletion mutants that exhibit an abnormal chromosome segregation pattern during meiosis also identified the sgol/SGO1 gene in fission yeast and budding yeast [Marston et al., 2004; Rabitsch et al., 2004]. Both yeasts depleted of Sgol cannot retain pericentromeric Rec8 during meiosis I, resulting in random chromosome segregation at meiosis II [Katis et al., 2004; Kitajima et al., 2004; Marston et al., 2004; Rabitsch et al., 2004]. Based on sequence homology restricted to a coiledcoil region at the $\mathrm{N}$-terminus and a cluster of basic residues at the C-terminus, shugoshin homologues have been identified in most eukaryotes from yeast to humans [Kitajima et al., 2004; Rabitsch et al., 2004]. Such homology searches identified the Drosophila MEI-S332 protein, which had previously been shown to be required for the persistence of centromeric cohesion during meiosis I [Kerrebrock et al., 1992, 1995], indicating that shugoshin/ MEI-S332 function in the protection of centromeric cohesion is conserved. It should be noted that although budding yeast and Drosophila contain a single shugoshin protein (Sgo1/MEI-S332) responsible for the protection of meiotic centromere cohesion [Kerrebrock et al., 1995; Kitajima et al., 2004], most other organisms including fission yeast, plants and mammals contain 2 shugoshin-like proteins (Sgo1 and Sgo2). In fission yeast and plants Sgo1 is predominantly responsible for the protection of centromeric cohesion during meiosis [Kitajima et al., 2004; Hamant et al., 2005], whereas Sgo2 carries out this function in mammals [Lee et al., 2008; Llano et al., 2008]. A further conserved feature of shugoshin/MEI-S332 proteins is their localisation at pericentromeres during meiosis I, consistent with their role in protecting cohesion in this region [Kerrebrock et al., 1995; Katis et al., 2004; Kitajima et al., 2004; Marston et al., 2004; Rabitsch et al., 2004; Hamant et al., 2005; Kiburz et al., 2005; Gomez et al., 2007; Lee et al., 2008].

\section{Shugoshin-PP2A-B' Prevents Cohesin Phosphorylation}

How does shugoshin prevent Rec 8 cleavage at the centromere during meiosis I? An important insight came from the finding that protein phosphatase $2 \mathrm{~A}(\mathrm{PP} 2 \mathrm{~A})$ is a major binding partner of budding and fission yeast meiotic Sgo1 [Kitajima et al., 2006; Riedel et al., 2006]. PP2A is a serine/threonine phosphatase that is composed of a heterotrimeric complex of scaffold, regulatory and catalytic subunits. Although several sub-families of PP2A regulatory subunits exist $\left(\mathrm{B}, \mathrm{B}^{\prime}, \mathrm{B}^{\prime \prime}\right.$ or $\left.\mathrm{B}^{\prime \prime \prime}\right)$ [Janssens and Goris, 2001; Lechward et al., 2001], meiotic Sgol co-purifies only with the subset of PP2A that contain B' regulatory subunits [Kitajima et al., 2006; Riedel et al., 2006]. PP2A complexes are found in many places in the cell; however, PP2A-B' preferentially co-localises with Sgol at meiotic centromeres [Kitajima et al., 2006; Riedel et al., 2006]. Sgol is required for the centromeric localisation of PP2A-B' in meiosis and, similar to sgol mutants, inactivation of PP2A-B' (by deletion of the catalytic subunit or the $\mathrm{B}^{\prime}$ subunit) causes premature loss of centromeric Rec8 [Kitajima et al., 2006; Riedel et al., 2006]. Similarly in mouse oocytes, the localisation of the PP2A catalytic subunit to centromeres depends on Sgo2 [Lee et al., 2008] and the treatment of oocytes with okadaic acid (a phosphatase inhibitor) induces premature separation of sister chromatids during meiosis I [Mailhes et al., 2003], suggesting that the protection of centromeric Rec 8 by shugoshin and PP2A might be a highly conserved mechanism. The artificial tethering of PP2A-B' to fission yeast chromosomes is sufficient to protect Rec8 from separase even in the absence of Sgol [Kitajima et al., 2006; Riedel et al., 2006], therefore the main role of shugoshin in Rec8 protection at meiotic centromeres appears to be to recruit PP2A-B' to that region. This idea is supported by a recent structural study of the human Sgol-PP2A interaction, which revealed that a shugoshin coiled-coil homodimer directly interacts with both the catalytic subunit and the $\mathrm{B}^{\prime}$ subunit of a single PP2A enzyme [Xu et al., 2009]. Mutation to alanine of 3 surface residues of budding yeast Sgol predicted to contact PP2A (sgo1-3A) completely abolishes the localisation of PP2A- $\mathrm{B}^{\prime}$ to the centromeres of meiotic cells, without affecting the centromeric localisation of Sgol itself [Xu et al., 2009]. The Sgo1-3A protein, although present at centromeres, fails to protect Rec8 at the centromere during meiosis I, thus providing strong evidence that the sole role of shugoshin in protecting centromeric Rec8 in meiosis is to recruit the phosphatase PP2A [Xu et al., 2009]. Similar mutations in mouse Sgo1 
also abolished its ability to recruit PP2A and prevent Rec8 removal from chromosomes when overexpressed in oocytes [Xu et al., 2009].

It is clear that shugoshin is required for the recruitment of PP2A- $\mathrm{B}^{\prime}$ to meiotic centromeres to protect Rec8, but what is the mechanism of PP2A- $\mathrm{B}^{\prime}$ action in this region? An early observation that the tethering of PP2A- $\mathrm{B}^{\prime}$ to chromosome arms in fission yeast not only prevents Rec8 removal from these regions, but also prevents Rec8 phosphorylation [Riedel et al., 2006], hinted that PP2A$\mathrm{B}^{\prime}$ might protect centromeric Rec8 by counteracting its phosphorylation. Indeed, initial phosphorylation site mapping of budding yeast Rec 8 found that Rec 8 phosphorylation at multiple sites is required for its efficient cleavage in meiosis I [Brar et al., 2006]. However, a caveat was that when 17 identified and predicted phosphorylation sites were mutated to prevent Rec 8 phosphorylation (rec8-17A), the observed delay in Rec8 cleavage in meiosis I was alleviated by depletion of Sgol [Brar et al., 2006]. This suggested that either Sgol-PP2A does not protect Rec8 solely by counteracting its phosphorylation, or that there are additional sites in Rec8 that must be phosphorylated for it to be cleaved. A recent re-examination of Rec8 phosphorylation sites identified additional serines and threonines within Rec8 that are phosphorylated in meiosis I [Katis et al., 2010]. When these additional sites, together with those previously identified [Brar et al., 2006], are mutated to generate an allele with 24 sites mutated (rec8-24A), a complete block to Rec8 cleavage is observed, which is not alleviated by depletion of Sgol [Katis et al., 2010]. Rec8-24A is also resistant to cleavage by separase in vitro and, crucially, mutating 14 of these sites to aspartic acid (rec8-14D) to mimic constitutive phosphorylation results in the premature loss of centromeric Rec8 in meiosis I, even though Sgol and PP2A-B' are present at the centromere [Katis et al., 2010]. Altogether this work now provides compelling evidence that the shugoshinPP2A complex protects centromeric Rec8 from separase by counteracting Rec 8 phosphorylation, thereby making it a poor substrate for cleavage.

\section{Multiple Kinases Promote Cohesin Cleavage}

Which kinases phosphorylate Rec 8 to promote its cleavage and are counteracted at the pericentromere by PP2A-B'? Three kinases, namely polo, casein kinase $1 \delta / \varepsilon$ (CK1) and the Dbf4-dependent Cdc7 kinase (DDK), have been implicated in this process. Budding yeast cells depleted of the polo-like kinase, Cdc5, show reduced Rec8 phosphorylation and are delayed in cleaving Rec8 in meiosis I [Clyne et al., 2003; Lee and Amon, 2003]. In addition, 11 Cdc5-dependent phosphorylation sites, and 4 further predicted Cdc5 consensus sites are among those substituted in Rec8-17A, the cleavage of which is delayed in meiosis I [Brar et al., 2006]. Furthermore, a phosphospecific antibody raised to one of the Cdc5-dependent sites recognises Rec 8 on chromosome arms, but not the centromeric Rec 8 that is retained beyond meiosis I, suggesting a possible role for $\mathrm{Cdc} 5$ in promoting Rec 8 cleavage [Brar et al., 2006]. Consistently, recombinant human polo-like kinase PLK1 also promotes Rec8 cleavage by separase in vitro [Kudo et al., 2009]. However, despite abundant evidence that polo-kinase phosphorylates Rec8, several observations indicated that Rec8 phosphorylation by polo kinase might not be essential for its cleavage. First, Rec8 cleavage is not prevented in Cdc5-depleted cells that lack Sgol [Brar et al., 2006]. Second, under certain mutant conditions, Rec8 is cleaved in prophase, even though Cdc5 is absent at this stage of meiosis [Oelschlaegel et al., 2005; Penkner et al., 2005]. Third, Cdc5 depletion retards the degradation of securin [Clyne et al., 2003; Lee and Amon, 2003], a separase inhibitor, and this may account for the delay in Rec8 cleavage [Katis et al., 2010].

In fact, 2 further kinases, CK1 and DDK, associate with Rec 8 from prophase onwards, and promote its cleavage in budding yeast [Katis et al., 2010]. The combined inactivation of CK1 and DDK caused a reduction in Rec8 phosphorylation and a block to Rec 8 cleavage similar to a non-phosphorylatable Rec8 mutant [Katis et al., 2010]. Conversely, the phospho-mimetic substituted Rec8 (Rec8-14D) partially restored Rec8 cleavage in cells where both CK1 and DDK were inactivated, indicating that these kinases enhance Rec 8 cleavage by promoting its phosphorylation [Katis et al., 2010]. Importantly, depletion of Sgol does not allow Rec8 cleavage in the absence of CK1 and DDK, implying that Sgol-PP2A-B' acts to counteract these kinases [Katis et al., 2010]. Similarly, CK1 was identified in a fission yeast screen for factors that promote Rec8 cleavage [Ishiguro et al., 2010]. Mutation of the putative CK1 phosphorylation sites on Rec8 causes a severe defect in its cleavage, although inactivation of CK1 results in only a modest delay to Rec8 cleavage during fission yeast meiosis [Ishiguro et al., 2010]. This suggests that an additional kinase(s) may act redundantly with CK1 to promote Rec 8 cleavage. This is unlikely to be polo, since depletion of the fission yeast pololike kinase Plo1 does not affect Rec8 phosphorylation or cleavage during meiosis and $\operatorname{Rec} 8$ is inefficiently phos- 


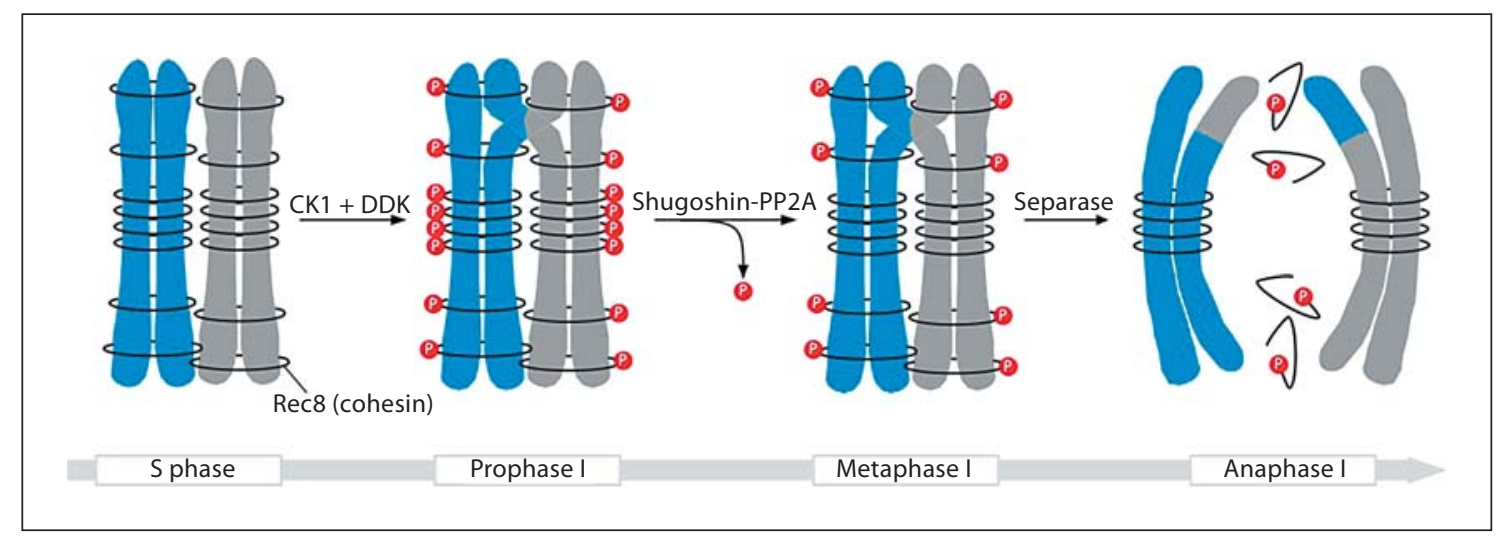

Fig. 2. A model for centromeric cohesion protection by shugoshin in meiosis I. During prophase I, multiple kinases phosphorylate Rec8 along the length of the chromosomes. Shugoshin recruits PP2A to centromeres where it locally dephosphorylates Rec8, rendering centromeric Rec 8 resistant to separase. In anaphase I, only the phosphorylated Rec8 is cleaved by separase, leading to homologous chromosome segregation. Centromeric Rec8 is retained to facilitate meiosis II chromosome segregation (adapted from Katis et al. [2010] and Ishiguro et al. [2010]). phorylated by Plo1 in vitro [Ishiguro et al., 2010]. However, intriguingly, the fission yeast Cdc7-related kinase, Hsk1, is required for efficient meiosis I nuclear division [Ogino et al., 2006] which could be consistent with a possible role for DDK in Rec8 cleavage, as in budding yeast.

The shugoshin-PP2A complex could protect centromeric Rec 8 by inhibiting the kinases responsible for Rec 8 phosphorylation, or by dephosphorylating Rec8 itself. In support of the latter possibility, immunoprecipitated PP2A-B' from fission yeast can efficiently dephosphorylate a Rec 8 fragment that was previously phosphorylated by CK1 in vitro [Ishiguro et al., 2010]. CK1 also localises to pericentromeric chromatin in fission yeast meiotic cells, the region where Rec 8 is enriched and protected by Sgol and, significantly, artificial tethering of excess CK1 to the pericentromere causes premature loss of centromeric Rec8 despite the presence of Sgol and PP2A-B' [Ishiguro et al., 2010]. Taken together, these results are consistent with a model whereby shugoshin-PP2A complexes protect Rec 8 by removing the phosphate groups added by CK1 (and potentially DDK) that are essential for Rec8 cleavage (fig. 2).

\section{Additional Functions of Shugoshin}

In addition to regulating the stepwise loss of cohesion during meiosis, shugoshin proteins have other functions that regulate chromosome segregation. In budding yeast and Drosophila, these multiple functions are carried out by a single shugoshin protein (Sgo1 and MEI-S332, respectively), whereas in most other eukaryotes, these functions are shared between 2 distinct shugoshin proteins referred to as Sgol and Sgo2. During vertebrate mitosis, Sgol is required to protect centromere cohesion, but in this case by counteracting cohesin dissociation that occurs during prophase rather than cohesin cleavage by separase as in meiosis [Salic et al., 2004; Tang et al., 2004; Kitajima et al., 2005; McGuinness et al., 2005]. This function of shugoshin may also depend on the recruitment of PP2A-B' to centromeres to dephosphorylate cohesin, although in this case the SA 2 cohesin subunit appears to be the relevant target [Hauf et al., 2005; McGuinness et al., 2005; Kitajima et al., 2006; Tang et al., 2006]. Budding yeast Sgol and human and fission yeast Sgo2 are also required during mitosis, where they function to ensure tension-generating bi-orientation of sister chromatids in metaphase [Indjeian et al., 2005; Huang et al., 2007; Kawashima et al., 2007; Vanoosthuyse et al., 2007]; however, their mechanisms of action appear to be quite distinct. Fission yeast Sgo2 is required for the full localisation of the chromosomal passenger complex (CPC) at centromeres [Kawashima et al., 2007; Vanoosthuyse et al., 2007], which play an essential role in the bi-orientation process [Ruchaud et al., 2007]. Human and mouse Sgo2 on the other hand localise both PP2A [Kitajima et al., 2006; Tanno et al., 2010] and mitotic centromere-associated kinesin (MCAK) [Huang et al., 2007; Llano et al., 2008] to the centromere, where the latter might be required to depolymerise incorrectly oriented kinetochore microtubules. 
Finally, the mitotic role of budding yeast Sgol appears to require an alternative subset of $\mathrm{PP} 2 \mathrm{~A}$ containing the $\mathrm{B}$ regulatory subunit (PP2A-B) to prevent premature separase activation when sister chromatids are not under tension [Clift et al., 2009]. Shugoshin proteins also play an additional role in meiotic chromosome segregation that is independent of Rec8 protection. Fission yeast sgo $2 \mathrm{mu}-$ tants and budding yeast SGO1 mutants have a modest increase in homologous chromosome missegregation during meiosis I [Katis et al., 2004; Kitajima et al., 2004; Marston et al., 2004; Rabitsch et al., 2004]. In fission yeast, this defect is likely explained by a reduction in CPC proteins at the centromere [Kawashima et al., 2007]; however, there are conflicting reports as to whether CPC localisation depends on Sgol in budding yeast meiosis [Yu and Koshland, 2007; Kiburz et al., 2008].

\section{Positive Regulation of Shugoshin}

How is shugoshin regulated? In budding and fission yeast, the spindle checkpoint component Bubl is required for the protection of centromeric cohesion at meiosis I [Bernard et al., 2001; Riedel et al., 2006]. Because the centromeric localisation of Sgol is abolished in yeast meiotic cells lacking Bub1 [Kitajima et al., 2004; Kiburz et al., 2005; Riedel et al., 2006], it is likely that the meiotic cohesion defect of bub1 mutants is due to the loss of Sgol from the centromere. Human Sgol and Sgo2, and mouse Sgo1 also depend on Bubl for centromere localisation [Tang et al., 2004; Kitajima et al., 2005; Huang et al., 2007; Perera and Taylor, 2010], indicating that the dependency of shugoshin on Bubl is highly conserved. Bubl knockout mouse oocytes, however, show only a partial centromere cohesion defect, suggesting that there might also exist Bub1-independent mechanisms for centromere protection in this organism [McGuinness et al., 2009]. The role of Bubl in localising shugoshin appears to be separate from its role as a spindle checkpoint component, as inactivation of the Bubl kinase domain does not affect the spindle checkpoint response to lack of attachment, yet centromeric shugoshin is lost [Fernius and Hardwick, 2007; Kawashima et al., 2010; Perera and Taylor, 2010]. Accordingly, budding and fission yeast Bubl kinase mutants show mitotic and meiotic defects identical to shugoshin mutants [Fernius and Hardwick, 2007; Kawashima et al., 2010]. Bubl was recently shown to phosphorylate a conserved serine residue at position 121 of histone $\mathrm{H} 2 \mathrm{~A}$, which is essential for the centromeric localisation and function of Sgol and Sgo2 in fission yeast mitosis and meiosis [Kawashima et al., 2010]. Furthermore, Bub1 phosphorylation of $\mathrm{H} 2 \mathrm{~A}$ is crucial for the centromeric localisation of Sgol in budding yeast and human cells, demonstrating that Bubl controls shugoshin function by a highly conserved mechanism [Kawashima et al., 2010].

Several other factors are also involved in promoting shugoshin localisation and function at the centromere. In fission yeast, Drosophila and human cells, shugoshin/ MEI-S332 fails to accumulate at centromeres when components of the chromosomal passenger complex (CPC) are inactivated [Resnick et al., 2006; Huang et al., 2007; Kawashima et al., 2007; Vanoosthuyse et al., 2007; Tsukahara et al., 2010]. However, depletion of Ipl1 (a CPC component) causes only a mild defect in Sgol localisation in budding yeast [Monje-Casas et al., 2007; Yu and Koshland, 2007]. The ability of human and mouse Sgo2 to recruit PP2A and MCAK to centromeres is also dependent on phosphorylation of Sgo2 by aurora B [Tanno et al., 2010]. In addition, the localisation of fission yeast Sgol to pericentromeric heterochromatin in meiosis is dependent on a direct interaction with the heterochromatin protein HP1 (called Swi6 in fission yeast) [Yamagishi et al., 2008]. Human Sgol also depends on HP1 to localise to the centromere, suggesting a conserved role for heterochromatin in shugoshin recruitment [Yamagishi et al., 2008]. Although budding yeast cells lack pericentromeric heterochromatin, the recruitment of Sgol to the pericentromere in budding yeast meiosis appears to be at least partially dependent on 2 kinetochore proteins ( $\mathrm{Iml} 3$ and Chl4), a meiosis-specific factor Spo13 and on the cohesin Rec8 itself [Kiburz et al., 2005]. The pericentromeric recruitment of Sgol might also involve an interaction with histone $\mathrm{H} 3$, at least in mitotic cells [Luo et al., 2010]. How all these factors cooperate with Bubl to ensure proper shugoshin localisation and function is not known. However, in fission yeast, H2A-S121 phosphorylation and HP1 appear to act independently [Kawashima et al., 2010]. Furthermore, in mouse embryonic fibroblasts (MEFs), the mechanism of shugoshin localisation changes depending on the cell cycle stage: whereas HP1 is required for Sgol localisation to heterochromatin during G2, Bub1 kinase activity recruits Sgol to centromeres only during prophase [Perera and Taylor, 2010].

\section{Inactivation of Shugoshin}

In order for sister chromatids to separate in meiosis II and in mitosis, the protection of centromere cohesion by shugoshin must be turned off. In fission yeast meiosis, 
Sgol is lost from centromeres in anaphase I due to its anaphase promoting complex (APC)-dependent degradation [Kitajima et al., 2004]. However, budding yeast and Drosophila shugoshin/MEI-S332 is present at centromeres in meiosis II and does not leave this region until the onset of anaphase II [Kerrebrock et al., 1995; Katis et al., 2004; Marston et al., 2004]. In mitotic cells of most organisms studied, Sgol is degraded in anaphase, likely due to ubiquitination by the APC [Salic et al., 2004; Karamysheva et al., 2009]. In contrast, the dissociation of Drosophila MEI-S332 from centromeres in meiosis II and anaphase of mitosis does not involve the APC, but instead requires phosphorylation of MEI-S332 by polo-kinase [Clarke et al., 2005]. Nevertheless, despite the active degradation of shugoshin and/or dissociation of shugoshin from centromeres in various organisms, several lines of evidence suggest that these mechanisms are not the trigger for shugoshin inactivation. First, the ectopic expression and centromeric localisation of fission yeast Sgol in meiosis II does not block the separation of sister chromatids [Rabitsch et al., 2004; Gregan et al., 2008]. Second, preventing MEI-S332 dissociation from centromeres does not prevent sister chromatid separation in Drosophila meiosis II and mitosis [Clarke et al., 2005]. Third, expression of a non-degradable Sgol does not interfere with sister chromatid separation or mitotic progression in human cells [Karamysheva et al., 2009].

It has been proposed that tension between sister chromatids is required to inactivate shugoshin in meiosis II and mitosis [Gomez et al., 2007; Lee et al., 2008]. In mouse oocytes, Sgo 2 co-localises with Rec 8 at the inner centromere in metaphase I, when centromeric Rec 8 is protected by Sgo 2 [Lee et al., 2008]. In metaphase II, however, when sister chromatids become bi-oriented and under tension, Sgo2 relocates toward the kinetochores away from Rec8, which remains at the inner centromere [Lee et al., 2008]. A similar tension-dependent re-distribution of Sgo 2 occurs in mouse spermatocytes in metaphase II [Gomez et al., 2007]. As Rec8 is no longer protected in meiosis II, these observations suggest that shugoshin relocation away from Rec8 upon tension might contribute to the deprotection of Rec8. In support of this view, fission yeast mutants that bi-orient sister chromatids in meiosis I prematurely lose centromeric cohesion [Vaur et al., 2005]. On the contrary, however, sister chromatids that bi-orient during meiosis I in budding yeast monopolin mutants retain centromere cohesion until meiosis II in an Sgoldependent manner [Petronczki et al., 2006]. Budding yeast might employ a different mechanism to inactivate shugoshin, although it is possible that the forced bi-ori- entation of sister chromatids in meiosis I might not reflect the proper tension-generating bi-orientation that would normally occur in meiosis II.

In human cells undergoing mitosis, both Sgol and Sgo2 also relocate from centromeres towards the kinetochores upon sister chromatid bi-orientation [Lee et al., 2008]. When these cells are arrested in metaphase by inhibition of the proteosome, tension-dependent shugoshin relocation towards kinetochores is accompanied by the loss of centromere cohesion, presumably because the prophase pathway is able to promote the dissociation of the now exposed centromeric cohesin [Lee et al., 2008]. This implies that tension between sister chromatids is sufficient to inactivate the protective function of shugoshin. Crucially, there is also evidence to suggest that tension between kinetochores is necessary for the inactivation of shugoshin. Human cells treated with the microtubule depolymerising drug nocodazole arrest in metaphase due to spindle checkpoint activation. Inhibition of the spindle checkpoint in these nocodazole-treated cells allows entry into anaphase without microtubules and therefore without tension between sister chromatids [Lee et al., 2008]. Remarkably, in such a 'tension-less' anaphase, both shugoshin and cohesin are retained at the centromere and co-localise with each other, indicating that tension-dependent re-distribution of shugoshin away from cohesin is necessary to inactivate shugoshin function [Lee et al., 2008]. At the molecular level, tension between sister kinetochores might also lead to the disassembly of the shugoshin-PP2A complex, perhaps by disrupting the shugoshin coiled-coil dimer that is required for PP2A binding [Xu et al., 2009].

\section{Conclusions}

The precise control of chromosome segregation during meiosis is of vital importance for sexually reproducing organisms. Key to this process is the shugoshin protein, which, from yeast to mammals, ensures the stepwise removal of sister chromatid cohesion during meiosis that is necessary for accurate reductional chromosome segregation. Since its discovery, much progress has been made into elucidating the mechanism by which shugoshin protects centromere cohesion. It is now apparent that the major, if not only, role of shugoshin in cohesion protection during meiosis is to recruit a phosphatase, PP2A, to centromeres. Here PP2A protects centromeric cohesin from separase by removing the phosphate groups on Rec 8 produced by multiple kinases that are essential for Rec 8 
cleavage. Shugoshin itself is also subject to complex regulation. The localisation of shugoshin at centromeres during meiosis is essential for cohesion protection, and depends on the phosphorylation of a single residue on histone $\mathrm{H} 2 \mathrm{~A}$ by the checkpoint kinase Bub1. Additional factors also contribute to shugoshin localisation and activity, although subtle variations in the shugoshin regulatory network are apparent between different organisms. Finally, the cohesion protection function of shugoshin must be inactivated in meiosis II to allow the separation of sister chromatids. This appears to be achieved by the tension-dependent relocalisation of shugoshin away from Rec8 that occurs upon the bi-orientation of sister chromatids in meiosis II. In sum, studies on shugoshin in several different model systems have led to a greater under- standing of the mechanisms that control chromosome segregation during meiosis. The continued examination of these mechanisms will undoubtedly provide important insights into the events that lead to human disorders that arise as a result of aneuploid gametes.

\section{Acknowledgements}

We apologise to colleagues whose work is not cited directly due to space restrictions. We are grateful to members of the Marston laboratory for helpful discussions. Work in the Marston laboratory is supported by a Wellcome Trust Career Development Fellowship to A.L.M., the Scottish Universities Life Sciences Alliance (SULSA) and the EMBO Young Investigator Programme.

\section{References}

Bernard P, Maure JF, Javerzat JP: Fission yeast Bubl is essential in setting up the meiotic pattern of chromosome segregation. Nat Cell Biol 3:522-526 (2001)

- Brar GA, Kiburz BM, Zhang Y, Kim JE, White F, Amon A: Rec8 phosphorylation and recombination promote the step-wise loss of cohesins in meiosis. Nature 441:532-536 (2006).

- Buonomo SB, Clyne RK, Fuchs J, Loidl J, Uhlmann F, Nasmyth K: Disjunction of homologous chromosomes in meiosis I depends on proteolytic cleavage of the meiotic cohesin Rec8 by separin. Cell 103:387-398 (2000).

-Clarke AS, Tang TT, Ooi DL, Orr-Weaver TL: POLO kinase regulates the Drosophila centromere cohesion protein MEI-S332. Dev Cell 8:53-64 (2005).

Clift D, Bizzari F, Marston AL: Shugoshin prevents cohesin cleavage by PP2A(Cdc55)-dependent inhibition of separase. Genes Dev 23:766-780 (2009).

-Clyne RK, Katis VL, Jessop L, Benjamin KR, Herskowitz I, et al: Polo-like kinase Cdc5 promotes chiasmata formation and cosegregation of sister centromeres at meiosis I. Nat Cell Biol 5:480-485 (2003).

- Eijpe M, Offenberg H, Jessberger R, Revenkova E, Heyting C: Meiotic cohesin REC8 marks the axial elements of rat synaptonemal complexes before cohesins SMC1beta and SMC3. J Cell Biol 160:657-670 (2003).

-Fernius J, Hardwick KG: Bubl kinase targets Sgol to ensure efficient chromosome biorientation in budding yeast mitosis. PLoS Genet 3:e213 (2007).

-Gerton JL, Hawley RS: Homologous chromosome interactions in meiosis: diversity amidst conservation. Nat Rev Genet 6:477487 (2005).

\section{Gomez R, Valdeolmillos A, Parra MT, Viera A, Carreiro C, et al: Mammalian SGO2 appears at the inner centromere domain and redis- tributes depending on tension across centro- meres during meiosis II and mitosis. EMBO Rep 8:173-180 (2007).}

Gregan J, Rumpf C, Li Z, Cipak L: What makes centromeric cohesion resistant to separase cleavage during meiosis I but not during meiosis II? Cell Cycle 7:151-153 (2008).

Hamant O, Golubovskaya I, Meeley R, Fiume E, Timofejeva L, et al: A REC8-dependent plant Shugoshin is required for maintenance of centromeric cohesion during meiosis and has no mitotic functions. Curr Biol 15:948954 (2005).

Hassold T, Hall H, Hunt P: The origin of human aneuploidy: where we have been, where we are going. Hum Mol Genet 16 Spec No 2:R203-R208 (2007).

-Hauf S, Roitinger E, Koch B, Dittrich CM, Mechtler K, Peters JM: Dissociation of cohesin from chromosome arms and loss of arm cohesion during early mitosis depends on phosphorylation of SA2. PLoS Biol 3:e69 (2005).

-Huang H, Feng J, Famulski J, Rattner JB, Liu ST, et al: Tripin/hSgo2 recruits MCAK to the inner centromere to correct defective kinetochore attachments. J Cell Biol 177:413-424 (2007).

Indjeian VB, Stern BM, Murray AW: The centromeric protein Sgol is required to sense lack of tension on mitotic chromosomes. Science 307:130-133 (2005).

Ishiguro T, Tanaka K, Sakuno T, Watanabe Y: Shugoshin-PP2A counteracts casein-kinase1-dependent cleavage of Rec8 by separase. Nat Cell Biol 12:500-506 (2010).
Janssens V, Goris J: Protein phosphatase 2A: a highly regulated family of serine/threonine phosphatases implicated in cell growth and signalling. Biochem J 353:417-439 (2001).

-Karamysheva Z, Diaz-Martinez LA, Crow SE, Li $\mathrm{B}, \mathrm{Yu} \mathrm{H}$ : Multiple anaphase-promoting complex/cyclosome degrons mediate the degradation of human Sgol. J Biol Chem 284: 1772-1780 (2009).

Katis VL, Galova M, Rabitsch KP, Gregan J, Nasmyth K: Maintenance of cohesin at centromeres after meiosis I in budding yeast requires a kinetochore-associated protein related to MEI-S332. Curr Biol 14:560-572 (2004).

Katis VL, Lipp JJ, Imre R, Bogdanova A, Okaz E, et al: Rec8 phosphorylation by casein kinase 1 and Cdc7-Dbf4 kinase regulates cohesin cleavage by separase during meiosis. Dev Cell 18:397-409 (2010).

Kawashima SA, Tsukahara T, Langegger M, Hauf S, Kitajima TS, Watanabe Y: Shugoshin enables tension-generating attachment of kinetochores by loading Aurora to centromeres. Genes Dev 21:420-435 (2007).

- Kawashima SA, Yamagishi Y, Honda T, Ishiguro $\mathrm{K}$, Watanabe Y: Phosphorylation of $\mathrm{H} 2 \mathrm{~A}$ by Bub1 prevents chromosomal instability through localizing shugoshin. Science 327: 172-177 (2010).

Kerrebrock AW, Miyazaki WY, Birnby D, OrrWeaver TL: The Drosophila mei-S332 gene promotes sister-chromatid cohesion in meiosis following kinetochore differentiation. Genetics 130:827-841 (1992).

- Kerrebrock AW, Moore DP, Wu JS, Orr-Weaver TL: Mei-S332, a Drosophila protein required for sister-chromatid cohesion, can localize to meiotic centromere regions. Cell 83:247-256 (1995). 
-Kiburz BM, Reynolds DB, Megee PC, Marston $\mathrm{AL}$, Lee $\mathrm{BH}$, et al: The core centromere and Sgol establish a 50-kb cohesin-protected domain around centromeres during meiosis I. Genes Dev 19:3017-3030 (2005).

Kiburz BM, Amon A, Marston AL: Shugoshin promotes sister kinetochore biorientation in Saccharomyces cerevisiae. Mol Biol Cell 19: 1199-1209 (2008).

Kitajima TS, Miyazaki Y, Yamamoto M, Watanabe Y: Rec8 cleavage by separase is required for meiotic nuclear divisions in fission yeast. EMBO J 22:5643-5653 (2003).

Kitajima TS, Kawashima SA, Watanabe Y: The conserved kinetochore protein shugoshin protects centromeric cohesion during meiosis. Nature 427:510-517 (2004).

Kitajima TS, Hauf S, Ohsugi M, Yamamoto T, Watanabe Y: Human Bubl defines the persistent cohesion site along the mitotic chromosome by affecting Shugoshin localization. Curr Biol 15:353-359 (2005).

-Kitajima TS, Sakuno T, Ishiguro K, Iemura S, Natsume T, et al: Shugoshin collaborates with protein phosphatase $2 \mathrm{~A}$ to protect cohesin. Nature 441:46-52 (2006).

Klein F, Mahr P, Galova M, Buonomo SB, Michaelis $\mathrm{C}$, et al: A central role for cohesins in sister chromatid cohesion, formation of axial elements, and recombination during yeast meiosis. Cell 98:91-103 (1999).

-Kudo NR, Wassmann K, Anger M, Schuh M, Wirth KG, et al: Resolution of chiasmata in oocytes requires separase-mediated proteolysis. Cell 126:135-146 (2006).

-Kudo NR, Anger M, Peters AH, Stemmann O, Theussl HC, et al: Role of cleavage by separase of the Rec8 kleisin subunit of cohesin during mammalian meiosis I. J Cell Sci 122: 2686-2698 (2009).

-Lechward K, Awotunde OS, Swiatek W, Muszynska G: Protein phosphatase 2A: variety of forms and diversity of functions. Acta Biochim Pol 48:921-933 (2001).

Lee BH, Amon A: Role of Polo-like kinase CDC5 in programming meiosis I chromosome segregation. Science 300:482-486 (2003).

Lee J, Kitajima TS, Tanno Y, Yoshida K, Morita $\mathrm{T}$, et al: Unified mode of centromeric protection by shugoshin in mammalian oocytes and somatic cells. Nat Cell Biol 10:42-52 (2008).

Llano E, Gomez R, Gutierrez-Caballero C, Herran Y, Sanchez-Martin M, et al: Shugoshin-2 is essential for the completion of meiosis but not for mitotic cell division in mice. Genes Dev 22:2400-2413 (2008)

Luo J, Xu X, Hall H, Hyland EM, Boeke JD, et al: Histone $\mathrm{H} 3$ exerts a key function in mitotic checkpoint control. Mol Cell Biol 30:537549 (2010)

-Mailhes JB, Hilliard C, Fuseler JW, London SN: Okadaic acid, an inhibitor of protein phosphatase 1 and $2 \mathrm{~A}$, induces premature separation of sister chromatids during meiosis I and aneuploidy in mouse oocytes in vitro. Chromosome Res 11:619-631 (2003).
Marston AL, Amon A: Meiosis: cell-cycle controls shuffle and deal. Nat Rev Mol Cell Biol 5:983-997 (2004).

- Marston AL, Tham WH, Shah H, Amon A: A genome-wide screen identifies genes required for centromeric cohesion. Science 303:1367-1370 (2004).

- McGuinness BE, Hirota T, Kudo NR, Peters JM, Nasmyth K: Shugoshin prevents dissociation of cohesin from centromeres during mitosis in vertebrate cells. PLoS Biol 3:e86 (2005).

McGuinness BE, Anger M, Kouznetsova A, GilBernabe AM, Helmhart W, et al: Regulation of APC/C activity in oocytes by a Bubl-dependent spindle assembly checkpoint. Curr Biol 19:369-380 (2009).

-Monje-Casas F, Prabhu VR, Lee BH, Boselli M, Amon A: Kinetochore orientation during meiosis is controlled by Aurora B and the monopolin complex. Cell 128:477-490 (2007).

Nasmyth K: Disseminating the genome: joining, resolving, and separating sister chromatids during mitosis and meiosis. Annu Rev Genet 35:673-745 (2001).

Nasmyth K, Haering CH: Cohesin: its roles and mechanisms. Annu Rev Genet 43:525-558 (2009).

-Oelschlaegel T, Schwickart M, Matos J, Bogdanova A, Camasses A, et al: The yeast APC/C subunit Mnd2 prevents premature sister chromatid separation triggered by the meiosis-specific APC/C-Ama1. Cell 120:773-788 (2005).

- Ogino K, Hirota K, Matsumoto S, Takeda T, Ohta $\mathrm{K}$, et al: Hsk1 kinase is required for induction of meiotic dsDNA breaks without involving checkpoint kinases in fission yeast. Proc Natl Acad Sci USA 103:8131-8136 (2006).

Parisi S, McKay MJ, Molnar M, Thompson MA, van der Spek PJ, et al: Rec8p, a meiotic recombination and sister chromatid cohesion phosphoprotein of the Rad21p family conserved from fission yeast to humans. Mol Cell Biol 19:3515-3528 (1999).

- Pasierbek P, Jantsch M, Melcher M, Schleiffer A, Schweizer D, Loidl J: A Caenorhabditis elegans cohesion protein with functions in meiotic chromosome pairing and disjunction. Genes Dev 15:1349-1360 (2001).

Penkner AM, Prinz S, Ferscha S, Klein F: Mnd2, an essential antagonist of the anaphase-promoting complex during meiotic prophase. Cell 120:789-801 (2005).

Perera D, Taylor SS: Sgol establishes the centromeric cohesion protection mechanism in G2 before subsequent Bub1-dependent recruitment in mitosis. J Cell Sci 123:653-659 (2010).

Petronczki M, Matos J, Mori S, Gregan J, Bogdanova A, et al: Monopolar attachment of sister kinetochores at meiosis I requires casein kinase 1. Cell 126:1049-1064 (2006).
Rabitsch KP, Gregan J, Schleiffer A, Javerzat JP, Eisenhaber F, Nasmyth K: Two fission yeast homologs of Drosophila Mei-S332 are required for chromosome segregation during meiosis I and II. Curr Biol 14:287-301 (2004).

- Rajagopalan H, Lengauer C: Aneuploidy and cancer. Nature 432:338-341 (2004).

Resnick TD, Satinover DL, MacIsaac F, Stukenberg PT, Earnshaw WC, et al: INCENP and Aurora B promote meiotic sister chromatid cohesion through localization of the Shugoshin MEI-S332 in Drosophila. Dev Cell 11: 57-68 (2006).

Riedel CG, Katis VL, Katou Y, Mori S, Itoh T, et al: Protein phosphatase $2 \mathrm{~A}$ protects centromeric sister chromatid cohesion during meiosis I. Nature 441:53-61 (2006).

Ruchaud S, Carmena M, Earnshaw WC: Chromosomal passengers: conducting cell division. Nat Rev Mol Cell Biol 8:798-812 (2007).

- Salic A, Waters JC, Mitchison TJ: Vertebrate shugoshin links sister centromere cohesion and kinetochore microtubule stability in mitosis. Cell 118:567-578 (2004)

Tang Z, Sun Y, Harley SE, Zou H, Yu H: Human Bubl protects centromeric sister-chromatid cohesion through Shugoshin during mitosis. Proc Natl Acad Sci USA 101:18012-18017 (2004).

Tang Z, Shu H, Qi W, Mahmood NA, Mumby $\mathrm{MC}, \mathrm{Yu} \mathrm{H}$ : PP2A is required for centromeric localization of Sgol and proper chromosome segregation. Dev Cell 10:575-585 (2006).

- Tanno Y, Kitajima TS, Honda T, Ando Y, Ishiguro K, Watanabe Y: Phosphorylation of mammalian Sgo2 by Aurora B recruits PP2A and MCAK to centromeres. Genes Dev 24: 2169-2179 (2010).

-Tsukahara T, Tanno Y, Watanabe Y: Phosphorylation of the CPC by Cdk1 promotes chromosome bi-orientation. Nature 467:719-723 (2010).

-Vanoosthuyse V, Prykhozhij S, Hardwick KG: Shugoshin 2 regulates localization of the chromosomal passenger proteins in fission yeast mitosis. Mol Biol Cell 18:1657-1669 (2007).

- Vaur S, Cubizolles F, Plane G, Genier S, Rabitsch PK, et al: Control of Shugoshin function during fission-yeast meiosis. Curr Biol 15:22632270 (2005).

Watanabe Y, Nurse P: Cohesin Rec8 is required for reductional chromosome segregation at meiosis. Nature 400:461-464 (1999).

- Xu Z, Cetin B, Anger M, Cho US, Helmhart W, et al: Structure and function of the PP2Ashugoshin interaction. Mol Cell 35:426-441 (2009).

- Yamagishi Y, Sakuno T, Shimura M, Watanabe $\mathrm{Y}$ : Heterochromatin links to centromeric protection by recruiting shugoshin. Nature 455:251-255 (2008).

-Yu HG, Koshland D: The Aurora kinase Ipl1 maintains the centromeric localization of PP2A to protect cohesin during meiosis. J Cell Biol 176:911-918 (2007). 